\title{
Schmidt CCD Astronomy at BAO
}

\author{
Jian-Sheng Chen \\ Beijing Astronomical Observatory, Chinese Academy of Sciences, \\ BEIJING 100080, CHINA
}

\begin{abstract}
Current users only use CCDs to cover a small fraction of the Schmidt fields. We discuss what kind of Schmidt astronomy is more efficient and the advantages of Schmidt telescopes over reflecting telescopes. The BAO CCD multi-object light curve survey system and the BATC 15 colour survey are introduced.
\end{abstract}

\section{Introduction}

A CCD has been used as the prime focus detector of the BAO $60 / 90 \mathrm{~cm} \mathrm{f/3}$ Schmidt telescope since 1988. The first CCD we used was the Thomson 576 $\times 384$ CCD with a pixel size of 23 micron. It was replaced in 1991 by a Ford Aerospace $2048 \times 2048 \mathrm{CCD}$ with a pixel size of 15 micron. The pixel scale is 1.67 arcsec and the field covered is about one square degree. The system noise is $6 \mathrm{e}^{-}$with two readout amplifiers. The fastest mode takes one minute to read out an entire CCD frame of 8 MBytes data. A typewriter-type filter arm device was built which can keep up to 20 filters on line for quick filter exchange, or for multi-colour observations without light obstruction.

\section{Strategy}

The unique advantage of the Schmidt telescope over reflectors is the wide field, which can be as large as 6 degrees for most existing Schmidt telescopes. With the rapid development of CCD techniques, especially large format and mosaic CCDs, it is possible to use CCDs to cover the whole Schmidt field provided by the Schmidt optics. However, current Schmidt CCD users, including the Kiso Schmidt mosaic CCD project, only use a small fraction of the available field. We ask the question: What kind of BETTER Schmidt astronomy could be achieved when a CCD is used as the Schmidt prime focus detector? (We do not discuss CCDs as detectors for Schmidt fibre optics, which take photometry and spectra of images selected from the whole Schmidt field). The question has two aspects: comparing the CCD with photographic emulsion and comparing Schmidt telescopes with reflector telescopes.

The "observation efficiency" of the Schmidt prime focus detector could be described by a quantity $\eta$ which is a function of

$$
\eta=f(S, d S, Q, d T, D R)
$$


Here, $S$ is the field covered by the detector, $d S$ is the spatial resolution determined by the pixel scale, $Q$ is the quantum efficiency, $d T$ is the waiting time defined as the minimum time between the end of one exposure to the start of the next exposure ( $d T$ determines the time resolution for short exposures), and $D R$ is the dynamic range. Larger pixel sizes allow larger CCD fields but with poorer spatial resolution for sites with good seeing conditions. CCDs are therefore more efficiently used at sites with average seeing conditions and small to intermediate aperture Schmidts. The BAO $60 / 90 \mathrm{~cm} \mathrm{Schmidt} \mathrm{is} \mathrm{located} \mathrm{at} \mathrm{a} \mathrm{site}$ with an average seeing disc of 2.5 arcsec. The sampling of $1.67 \mathrm{arcsec} / \mathrm{pixel}$ is reasonable with slight difficulty in defining the point spread function. With this spatial resolution it is better suited for work on high galactic latitude regions and on spatially extended objects such as nearby galaxies. The BAO CCD field is still only a small fraction of the Schmidt field. So it is better to work on selected areas and is not suitable for all sky surveys. The UV-coated thick CCD has a quantum efficiency which is only a factor of 3 to 4 better than IIIa-J type emulsion at blue wavelengths, but is more than an order of magnitude better than the emulsion at red and near-infrared wavelengths. It is therefore more efficient to work at red colours, for example, for high redshift QSO and brown dwarf surveys. The waiting times for photographic observations are typically a few minutes to change the photographic plate and the filter to start the next exposure. The waiting time, $d S$, for the CCD is determined by the readout time, which is about 1-2 minutes for $2048 \times 2048$ pixels, not much gain compared with photographic observations. However, if the user is interested in selected objects in the field, then multi-window techniques can reduce the readout time by more than a factor of 10 . So the CCD is suitable to monitor/discover and to study short period variable objects.

What is the advantage of using a CCD with a Schmidt telescope, rather than a cheaper relecting telescope, which has a flat focal plane? The advantage, from our experience, is that with Schmidt optics, one can get very good dome flats by using a diffuser in front of the corrector. We found that the so-called super-sky flat field is not only signal-to-noise limited, but also no longer flat for sky areas of one degree or more. The twilight flat field is even worse with gradients as large as a few per cent. Dome flat fields can provide images with signal-to-noise values as high as needed. A CCD image obtained at the telescope focal plane for a uniform light source at infinity (e.g., from the sky) is equal to that for a nearby isotropic light source, if the integration areas for the different directions of light are the same. This can be achieved if the diffuser is placed at the position of the entrance aperture, which is the corrector plate for Schmidt optics. To get a highly isotropic light distribution is much easier than an uniform light source. Also, the Schmidt telescope can easily avoid the scattered light which deforms the flat field structure.

\section{The BAO $60 / 90 \mathrm{~cm}$ Schmidt Multi-object Light Curve Survey System}

We are using a multi-window technique to read out only those parts of the CCD which are occupied by the stars we are interested in. Each star is covered by a window, the size of which can be changed according to the seeing conditions. 
The readout time and disc space required are then much smaller. A single 8 $\times 512 \times 512$ bits image board is used to give real-time monitoring of the light curves. The upper $8 \times 125 \times 512$ bits image board are used to store up to 125 light curves with 8 bits for brightness and 512 bits for timing. The remaining part of the image board is used for graphic display of the light curves in page mode. Stars brighter than $15 \mathrm{mag}$ have been observed with accuracy of 0.01 mag using a sampling time of 20s. Cataclysmic variables and other short period variables have been found (Wei et al. 1990)

\section{The BATC (Beijing-Arizona-Taipei-Connecticut) Colour Survey}

The goal of this survey is to make a comprehensive spectral and temporal study for 450 square degrees in 500 high galactic latitude fields in the sky, complete to $\mathrm{V}=21$ mag. Fifteen intermediate passband interference filters from $3200 \AA$ to about 1 micron are used to measure the spectral energy distribution (SED) of every object in these regions at constant $d z / z$. Of the 500 fields, 150 are chosen centered on QSOs that cover the full range of known QSO properties. These will provide examples of the SEDs of known types of QSOs to act as templates. In addition, 150 are chosen to be spiral galaxies 2-5 arcmin in diameter; 150 are chosen at random as control fields and 50 are chosen for calibration. From this survey, we expect to measure 10,000 QSOs and active galaxies, 100,000 normal galaxies and 100,000 stars. Multiple exposures in each filter will be taken with different time scales. The data will be used to search for variables and to improve the signal-to-noise for non-variables. The data will be used to address many extragalactic and galactic astrophysical problems : (a) find QSOs with redshift up to 6, (b) study the large scale distribution of QSOs in the universe, (c) determine the inter-relationships between the structure, stellar populations and interstellar media of nearby spiral galaxies; (d) study the spatial distribution and spectral evolution of galaxies between the present day and a redshift of $z=0.2-0.3$, and (e) determine the large scale topology of the universe. The comparable stars found can be used to study Galactic structure (Chen 1993).

\section{Acknowledgements}

The work of this paper is partially supported by a grant from the National Natural Science Foundation of China.

\section{References}

Chen J.-S., 1994, in Astronomy from Wide-Field Imaging, Proc. IAU Symp. 161 , in press

Wei M.-Z., Chen J.-S. \& Jian Z.-J., 1990, PASP102, 698 